\section{AB0241 1 FACTORS THAT DIFFERENTLY ASSOCIATE WITH THE RETENTION ON TARGETED THERAPY OF PATIENTS WITH RHEUMATOID ARTHRITIS}

E. Zhilyaev ${ }^{1,2}$, G. Lukina ${ }^{3}$, E. Koltsova ${ }^{3,4}$, K. Lytkina ${ }^{5}$, E. Shmidt ${ }^{6}{ }^{1}$ European $^{2}$ Medical Center, Medical Derection, Moscow, Russian Federation; ${ }^{2}$ Russian Medical Academy of Continous Professional Education, Rheumatology, Moscow, Russian Federation; ${ }^{3}$ A.S. Loginov Moscow Clinical Scientific Center, Rheumatology, Moscow, Russian Federation; ${ }^{4}$ Research Institute of the Organization of Health and Healthcare Managment, Organizational and Methodological Department of Rheumatology, Moscow, Russian Federation; ${ }^{5}$ City Clinical Hospital, Rheumatology, Moscow, Russian Federation;

${ }^{6}$ N.I.Pirogov City Clinical Hospital, Rheumatology, Moscow, Russian Federation

Background: A personalized approach to prescribing targeted drugs implies the availability of data that can be used to suggest that a particular drug is better suited for a given patient than others. Retention on the treatment can be considered as an integral indicator of the acceptability of the drug in real practice. For the purposes of treatment personalization, the indicators that are associated with better retention on some drugs and with worse retention, or that do not have a clear association with retention on others, are of particular interest.

Objectives: to identify predictors those are differently associated with retention on different targeted drugs for the treatment of rheumatoid arthritis.

Methods: Data of the patients with rheumatoid arthritis (RA) from the Moscow Unified Register of Arthritis (MUAR) were used. The analysis includes episodes of treatment with biological or synthetic targeted drugs (tDMARDs) that continue or end during the patient's follow-up in the registry. Within the framework of the Cox proportional risk regression model, significant independent predictors of tDMARDs cancellation were identified. These indicators were later considered as confounders. Further, in the generated linear regression risk model, all available indicators were tested for the presence of a statistically significant interaction with the factor of used tDMARD.

Results: The study included 944 episodes of tDMARDs treatment (Table 1.) in 832 patients. The average age is $55.3+12.4$ years. There were 131 males $(16.1 \%)$. The average duration of the disease is $13.1+9.4$ years. Smoking, family history of $\mathrm{RA}$, and the nature of RA onset (acute or gradual) were identified as reliable mutually independent predictors of retention on tDMARDs treatment. As a result of the search for indicators that reliably interact with used tDMARD, patient's reports of the association of the onset of arthritis 1) with symptoms of intestinal dyspepsia $(p<0.001), 2)$ with genital inflammatory disease $(p=0.002)$ were revealed. Most strongly associated with factor 1 ) was retention on abatacept (ABA), rituximab (RIT), and tofacitinib (TOFA). The second indicator was most strongly associated with retention on $\mathrm{ABA}$, adalimumab (ADA) and TOFA (Picture 1).

Table 1. Description of treatment episodes included in the analysis

\begin{tabular}{|c|c|c|c|c|c|c|c|c|c|c|}
\hline & ABA & $A D A$ & CER & ETAT & GOL & INF & RIT & TOC & TOFA & Total \\
\hline $\begin{array}{l}\text { Number of } \\
\text { episodes }\end{array}$ & 175 & 150 & 34 & 186 & 8 & 55 & 130 & 113 & 93 & 944 \\
\hline Completed & 39 & 51 & 13 & 55 & 2 & 39 & 31 & 14 & 9 & 253 \\
\hline \multicolumn{10}{|c|}{ Line of treatment } & \\
\hline 1 & 83 & 24 & 113 & 120 & 3 & 50 & 70 & 66 & 35 & 564 \\
\hline & $(47,4 \%)$ & $(70,6 \%)$ & $(60,8 \%)$ & $(80,0 \%)$ & $(37,5 \%)$ & $(90,9 \%)$ & $(61,9 \%)$ & $(50,8 \%)$ & $(37,6 \%)$ & $(59,7 \%)$ \\
\hline 2 & 62 & 6 & 52 & 24 & 2 & 4 & 22 & 39 & 30 & 241 \\
\hline & $(35,4 \%)$ & $(17,6 \%)$ & $(28,0 \%)$ & $(16,0 \%)$ & $(25,0 \%)$ & $(7,3 \%)$ & $(19,5 \%)$ & $(30,0 \%)$ & $(32,3 \%)$ & ) $(25,5 \%)$ \\
\hline 3 & 22 & 1 & 13 & 6 & 1 & 1 & 15 & 21 & 14 & 94 \\
\hline & $(12,6 \%)$ & $(2,9 \%)$ & $(7,0 \%)$ & $(4,0 \%)$ & $(12,5 \%)$ & $(1,8 \%)$ & $(13,3 \%)$ & $(16,2 \%)$ & $(15,1 \%)$ & ) $(10,0 \%)$ \\
\hline 3 & 8 & 3 & 8 & 0 & 2 & 0 & 6 & 4 & 14 & 45 \\
\hline & $(4,6 \%)$ & $(8,8 \%)$ & $(4,3 \%)$ & $(0,0 \%)$ & $(25,0 \%)$ & $(0,0 \%)$ & $(5,3 \%)$ & $(3,1 \%)$ & $(15,1 \%)$ & $(4,8 \%)$ \\
\hline
\end{tabular}

ABA - abatacept, ADA - adalimumab, CER - certolizumab paegol, ETA- etanercept, GOL - golimumab, INF - infliximab, TOC- tocilizumab, TOFA - tofacitinib.

Conclusion: Anamnestic indications of the association of the onset of rheumatoid arthritis with symptoms of intestinal dyspepsia and with preceding genital inflammation are differently associated with retention on different targeted drugs in patients with RA. These indicators can be used to personalize the treatment.
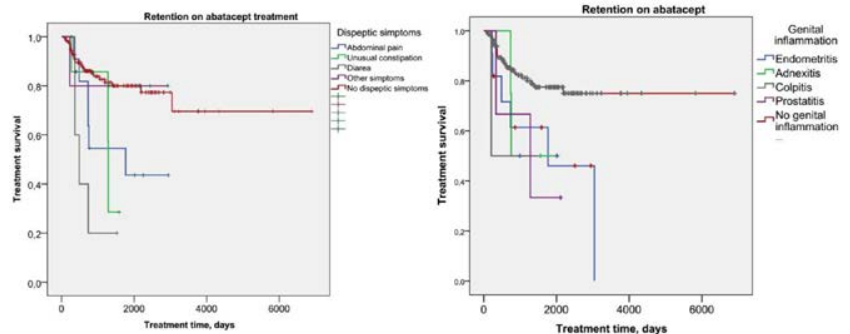

Picture 1. Retention on the abatacept depending on the features of the onset of rheumatoid arthritis
Disclosure of Interests: None declared

DOI: 10.1136/annrheumdis-2021-eular.3953

\section{AB0242 CERTOLIZUMAB IN MONOTHERAPY AS EFFECTIVE THAN IN COMBINATION IN RHEUMATOID ARTHRITIS PATIENTS}

P. Osorio $^{1}$, L. Villarreal ${ }^{1}$, W. Rivero ${ }^{2}$, L. Ibata ${ }^{3}$, S. Martinez ${ }^{3}$, A. Rojas-Villarraga ${ }^{4}$ P. Santos-Moreno ${ }^{5} .^{1}$ Biomab - Center for Rheumatoid Arthritis, Bogota,

Colombia, Administration, Bogota, Colombia; ${ }^{2}$ Biomab - Center for Rheumatoid Arthritis, Bogota, Colombia, Pharmaceutical Chemist, Bogota, Colombia; ${ }^{3}$ Epithink Health Consulting, Epidemiology, Bogota, Colombia; ${ }^{4}$ Fundacion Universitaria de Ciencias de la Salud - FUCS, Research Division, Bogota, Colombia; ${ }^{5}$ Biomab - Center for Rheumatoid Arthritis, Bogota, Colombia, Rheumatology, Bogota, Colombia

Background: Tumour necrosis factor-a (TNFa) inhibitors are one of the most used biological therapy for rheumatoid arthritis (RA) patients without response to conventional disease-modifying antirheumatic drugs (cDMARDs). There is scarce information about biological DMARDs (bDMARDs) effectiveness as monotherapy in real life.

Objectives: To assess the effectiveness of Certolizumab pegol in real-life clinical practice in a cohort of patients with RA and previous failure to cDMARDs who received certolizumab as monotherapy, combined with Methotrexate (MTX), combined with leflunomide (LFN), or both MTX and LFN.

Methods: A retrospective cohort study was conducted at a specialized center for RA management in Colombia. Patients treated with Certolizumab as monotherapy or in combination with MTX, or LFN, or both MTX plus LFN, (2012 - 2020) were included. The indication of certolizumab was a independent decision based on disease activity. Patients could be taking adjuvant medications for pain and/or corticosteroids. Certolizumab was administered $400 \mathrm{mg} \mathrm{SC}$ monthly after three induction doses at weeks 0,2 y 4 , as monotherapy or in combination with MTX (until $25 \mathrm{mg} /$ week), LFN ( $20 \mathrm{mg} /$ day) or both as a first-line treatment after failure with cDMARDs or as a second-line treatment after failure with one or more bDMARDs. Effectiveness was assessed at three, six, and twelve months according to the change in DAS28. Exploratory comparisons of numeric variable data between groups were done (ANOVA). Chi-square test and Fisher's exact test were used for categorical variables. Multivariate analyses (logistic regressions) were performed to analyse factors related to the response at 6 and 12 months.

Results: Of 181 enrolled patients, 24 received certolizumab as monotherapy, 62 combined with MTX, 47 in combination with LFN and 48 in combination with MTX and LFN. $55.2 \%$ of patients were first-line treatment, without significant differences between groups. Mean age of subjects included was $57.87 \pm 12.33$ with a mean age of around 60 years in patients treated with CERTO+MTX and CERTO+LFN $(p=0,014)$. Women predominated in all groups $(84 \%$ of the total) $(p=0,275)$. The duration of arthritis on average was $8.27 \pm 8.73$ years, without significant differences between treatment groups $(p=0,871)$. In terms of disease activity, $78.5 \%$ of patients were in moderate or high disease activity, according to DAS28, without significant differences between the groups $(p=0,787)$. Differences in the number of biologicals and corticoid use were observed, patients in the combination groups had used in a higher proportion two or more biological $(p=0,046)$ than in the monotherapy group and had used corticosteroids $(p=0,042)$ more frequently. Overall, there were decreased disease activity, at 3 and 6 months with no significant differences between groups $(p=0,08)$. At 12 months of treatment, there was a higher maintenance in mild activity/remission response in monotherapy group compared to the others $(p=0,01)$. In the multivariate analysis, no differences were observed in the response at 6 and 12 months between the treatment groups. The response at three months was the only variable associated with the 6-month response (OR 5.46; $\mathrm{Cl} 95 \% 2.08$ - 14.32). The response at three months (OR 4.04; $\mathrm{Cl}$ $95 \%$ 1.28-12.69) and positive anti-CCP (OR 3.83; Cl 95\% 1.11-13.21) were associated with 12-month response.

Conclusion: These exploratory results show a clear trend of Certolizumab being effective as monotherapy in patients previously treated with cDMARDs and even after being treated with one bDMARD. Although results are not statistically different, it seems that certolizumab in monotherapy could be as least as effective as combination therapy. Prospective studies with larger sample size and with a structured follow-up are needed to confirm these findings.

Disclosure of Interests: Paola Osorio: None declared, Laura Villarreal: None declared, Wilberto Rivero: None declared, Linda Ibata: None declared, Susan Martinez: None declared, Adriana Rojas-Villarraga: None declared, Pedro Santos-Moreno Speakers bureau: has received fees for conferences from: Abbvie, Abbott, Biopas-UCB, Bristol, Janssen, Pfizer, Roche, Sanofi, Consultant of: has received fees for counseling, advisory boards from: Abbvie, Abbott, BiopasUCB, Bristol, Janssen, Pfizer, Roche, Sanofi, Grant/research support from: has received fees for research grants from: Abbvie, Abbott, Biopas-UCB, Bristol, Janssen, Pfizer, Roche, Sanofi

DOI: 10.1136/annrheumdis-2021-eular.4008 